\title{
Antioxidant and Anti-Inflammatory Effects of Origanum majorana L. Methanolic Extract on Bile Duct Ligation in Male Rats
}

\author{
Izadpanah Gheitasi $\mathbb{D}^{1},{ }^{1}$ Nikta Motaghi $\mathbb{D}^{2},{ }^{2}$ Hossein Sadeghi $\mathbb{D},{ }^{1}$ Heibatollah Sadeghi $\mathbb{D},{ }^{1}$ \\ Zahra Moslemi $\mathbb{D}{ }^{2}{ }^{2}$ Mahdieh Eftekhari $\mathbb{D},{ }^{3}$ Nasrin Shakerinasab $\mathbb{D D}^{1},{ }^{1}$ \\ and Amir Hossein Doustimotlagh $\mathbb{D D}^{1}$
}

\author{
${ }^{1}$ Medicinal Plants Research Center, Yasuj University of Medical Sciences, Yasuj, Iran \\ ${ }^{2}$ Student Research Committee, Yasuj University of Medical Sciences, Yasuj, Iran \\ ${ }^{3}$ Department of Pharmacognosy and Pharmaceutical Biotechnology, Faculty of Pharmacy, \\ Kermanshah University of Medical Sciences, Kermanshah, Iran
}

Correspondence should be addressed to Amir Hossein Doustimotlagh; amirhosseindoustimotlagh@gmail.com Received 17 March 2021; Revised 7 April 2021; Accepted 30 April 2021; Published 7 May 2021

Academic Editor: Amin Tamadon

Copyright (c) 2021 Izadpanah Gheitasi et al. This is an open access article distributed under the Creative Commons Attribution License, which permits unrestricted use, distribution, and reproduction in any medium, provided the original work is properly cited.

\begin{abstract}
Introduction. Cholestasis is caused by malfunction of the hepatobiliary system. This disorder is the result of the accumulation of bile fatty acids and other toxins in the liver. The aim of the current study was to investigate the antioxidative and hepatoprotective effects of methanolic extract of Origanum majorana L. (OM) on hepatic disorder and tissue damage induced by bile duct ligation (BDL) in rats. Materials and methods. Twenty-eight male Wistar rats were randomly divided into 4 groups including sham control group received vehicle $(\mathrm{SC}-\mathrm{V})$, bile duct ligation received vehicle (BDL-V), bile duct ligation group received OM extract $(\mathrm{BDL}+\mathrm{OM})$, and sham control group received $\mathrm{OM}$ extract $(\mathrm{SC}+\mathrm{OM})$. One day after surgery, the animals received vehicle or methanolic extract of OM $300 \mathrm{mg} / \mathrm{kg} /$ day for 7 consecutive days by oral gavage. Finally, the animals were anesthetized and the blood samples were collected from each animal. After sacrificing of animals, liver tissue from each rat was removed and divided into three parts: one part was used for preparing of homogenized tissue, one part was fixed in $10 \%$ neutral formalin for histopathology examination, and the third part was kept in liquid nitrogen for gene expression analysis. Biomarkers of oxidative stress in the liver tissue and serum, as well as histopathological changes of the liver, were assessed. Also, the gene expression of IL1 , TNF- $\alpha$, TGF- $\beta$, and $\alpha$-SMA has been measured. Results. The results showed that BDL-V significantly increased the activity of ALT, AST, ALP, and total bilirubin compared to the SC-V group. The oxidative stress markers such as MDA and FRAP significantly increased due to BDL, while the CAT activity reduced in the BDL-V group compared to SC-V group. Oral treatment with OM reduced ALT and AST activity, although it was not statistically significant. OM treatment considerably increased the activity of CAT compared to BDL group. BDL-V induced a significant histological change in the liver, while treatment with OM at a dose of $300 \mathrm{mg} / \mathrm{kg}$ showed a minor effect on histopathological changes. In addition, the mRNA of IL- 1 , TNF- $\alpha$, TGF- $\beta$, and $\alpha$-SMA significantly increased in the BDL-V group, while treatment with OM only significantly reduced TGF- $\beta$ in comparison with BDL-V rats. Conclusions. The results of the present study showed that oral administration of OM extract had a moderate protective effect on cholestasis due to BDL. Indeed, more studies with different doses of extract are needed to confirm this finding.
\end{abstract}

\section{Introduction}

Liver is one of the main organs of the body that is involved in providing energy, excretion of harmful substances and drugs, and maintaining and regulating homeostasis [1,2]. Hepatic cholestasis is due to defect in the production, secretion, and flow of bile which causes the accumulation of bile acids in the liver. Bile acids accumulated in pathological concentrations cause the inflammation of liver tissue, destruction of hepatocytes, and the production of reactive oxygen species (ROS) and the development of oxidative stress $[1,3]$. Intrahepatic cholestasis can be inherited or 
acquired, such as drug-induced liver injury, pregnancy, hepatitis, and primary biliary cirrhosis. In untreated cases, hepatic cholestasis can cause itchy skin, hyperlipidemia, and jaundice, which progresses to liver cirrhosis, fibrosis, and liver failure. Ursodeoxycholic acid is currently the only Food and Drug Administration (FDA) approved drug for the treatment of cholestasis that has not seen improvement in cholestasis symptoms in some patients after taking this drug [4]. Despite liver disorders being a global problem with high mortality and morbidity, there is no effective treatment to control their progression. New drugs for the treatment of chronic liver disorders have also been found to have harmful effects [5]. The phenomenon of oxidative stress is due to the overproduction of ROS, which leads to an imbalance of antioxidant and pro-oxidative processes [6]. ROS contains a large group of molecules such as peroxyl radical, superoxide radical, nitric oxide, hypochlorous acid, hydroxyl radical, and hydrogen peroxide [7]. Antioxidants can produce harmless products by reacting with free radicals. Catalase (CAT), superoxide dismutase (SOD), glutathione reduced $(\mathrm{GSH})$, and glutathione peroxidase $(\mathrm{GPX})$ are a group of endogenous antioxidants [8]. Marjoram plant with the scientific name of Origanum majorana L (OM) belongs to the Lamiaceae family and grows mostly in America, Africa, Asia, Europe, and Arabian Peninsula [9]. Marjoram leaves are used in cases such as asthma, nervous problems, insomnia, and digestive disorders [10]. Pharmacological research has shown that OM has antifungal, antioxidant, anti-spasmodic, analgesic, antibacterial, antimutagenic, antitumoral, antithrombin, and antihyperglycaemic effects [11]. Research also has shown that ursolic acid of OM and its essential oil, especially thymol and carvacrol, have antiviral and bactericidal, antiseptic, and antifungal properties [12]. Ursolic acid and rosmarinic acid, as the isolated compounds from OM, also have liver protective and fat reducing properties [1, 13]. Bile duct ligation is an in vitro model of hepatic cholestasis induction that causes secondary biliary cirrhosis and oxidative stress, which increases liver function markers in animal models $[14,15]$. Studies in $\mathrm{CCl}_{4}$-intoxicated rats have shown that methanolic extract of $\mathrm{OM}$ significantly reduces aspartate aminotransferase (AST), alanine aminotransferase (ALT), alkaline phosphatase (ALP), and its protective effect on the liver, possibly due to its inhibitory effect on cytochrome P450 and thus preventing the formation of free radicals [16]. The present study was designed to examine the effect of OM methanolic extract against oxidative damage, oxidative stress markers, and antioxidant status in BDL-induced hepatic injury in rats.

\section{Material and Methods}

2.1. Chemicals. Ethylenediaminetetraacetic acid (EDTA), pentobarbital, thiobarbituric acid (TBA), and 5,5'dithiols-(2-nitrobenzoic acid) (DTNB) were purchased from Sigma Chemical Co (St Louis, MO, USA). Trichloroacetic acid (TCA), 2,4-dinitrophenylhydrazine (DNPH), and formaldehyde were obtained from Merck (Germany).
2.2. Preparation of Origanum majorana Methanolic Extract. In the summer of 2020, fresh aerial parts of Origanum majorana were collected from the foothills of Dena Mountain and then identified by the botanist (herbarium No: MPISB158).

Origanum majorana aerial parts were cleaned, then placed in room air, kept away from direct light for several days to dry, and then powdered. 100 grams of dried powder plant was soaked in $1000 \mathrm{ml}$ of $70 \%$ methanol (methanol: water $70: 30$ ) and was placed at $37^{\circ} \mathrm{C}$ for 48 hours. The resulting mixture was centrifuged and filtered through Whatman filter paper NO. 1; after that, it was concentrated as much as possible under a vacuum rotary apparatus. Then, the extract was then dried in an incubator at $40^{\circ} \mathrm{C}$ and stored in a freezer at $-20^{\circ} \mathrm{C}$ for the next experiments [17].

2.3. Animals and Experimental Design. In this study, 28 male Wister rats (3 months old, 200 to $250 \mathrm{~g}$ ) were purchased from Shahrekord University of Medical Sciences. The rats were kept in animal laboratory of Yasuj University of Medical Science under the 12-hour light/dark cycle condition, and they had free access to tap water and food (Ethical code; IR.YUMS.REC.1398.158).

Rats were randomly divided into 4 groups of 7 animals each as follows: group 1: sham control + vehicle (SC-V), in which surgery was performed but the bile duct was not closed; group 2: cholestatic group + vehicle (BDL-V), in which surgery was performed with bile duct closure; group 3: $\mathrm{SC}+\mathrm{OM}$ extract; group 4: BDL + OM. BDL was performed under antiseptic conditions and the animals received daily the methanolic extract of OM orally $300 \mathrm{mg} / \mathrm{kg}$ for 7 consecutive days. To do bile duct ligation on experimental groups, after induction of general anesthesia with ketamineHCL $50 \mathrm{mg} / \mathrm{kg}$ and xylazine-HCL $10 \mathrm{mg} / \mathrm{kg}$, the common bile duct of the animal was tied on two points and cut in half [18]. The day after surgery, groups 1 and 2 received normal saline and groups 3 and 4 received $300 \mathrm{mg} / \mathrm{kg} \mathrm{OM}$ for 7 consecutive days. One week later, animals were anesthetized with ether, and the blood was collected by puncturing the heart for biochemical tests. Liver tissue was removed after collecting the blood. Three parts of the liver tissue were allocated; one of them was kept for gene expression analysis in liquid nitrogen, one of them was stored for histological examination in 10 percent formalin, and the third section was homogenized $(10 \%, \mathrm{w} / \mathrm{v})$ in PBS $(10 \mathrm{mmol} / \mathrm{L}, \mathrm{pH} 7.4)$.

2.4. Biochemical Analysis. Plasma was separated by centrifugation at $3,000 \mathrm{rpm}$ at room temperature for $15 \mathrm{~min}$. The activity of liver enzymes including ALT, AST, and ALP, and the level of total protein and total bilirubin were measured colorimetry using Pars Azmoon kit.

2.5. Measurement of Hydroxyproline Content. The level of tissue hydroxyproline was determined based on previous study [1]. $0.1 \mathrm{~g}$ of liver tissue was hydrolyzed in $6 \mathrm{~N} \mathrm{HCl}$ for $18 \mathrm{~h}$, Chloramine-T reagent and citrate buffer were added; then, fresh Ehrlich's reagent ( $15 \mathrm{~g}$ of $\mathrm{p}$-dimethyl amino 
benzaldehyde in n-propanol/perchloric acid; $2: 1 \mathrm{v} / \mathrm{v})$ was added and placed in a warm water bath $\left(60^{\circ} \mathrm{C}\right)$ for $15 \mathrm{~min}$. Finally, the optical density was determined spectrophotometrically at $550 \mathrm{~nm}$.

\subsection{Oxidative Stress Markers}

2.6.1. Measurement of Ferric Reducing Antioxidant Power (FRAP). In this method, the ability of plasma and homogenized tissue to reduce ferric ions $\left(\mathrm{Fe}^{3+}\right)$ was measured. By reducing the ferric ions and converting them to $\mathrm{Fe}^{2+}$ at an acidic $\mathrm{pH}$ in the presence of TPTZ, the Fe-TPTZ complex was formed, which was blue. The resulting color intensity was measured spectrophotometrically at wavelength of $593 \mathrm{~nm}$ [19].

2.6.2. Measurement of Malondialdehyde. The concentration of malondialdehyde (MDA) in the homogenized tissue and plasma was measured after reaction with thiobarbituric acid. The optical absorption of the red product was measured at $535 \mathrm{~nm}[20]$.

2.6.3. Measurement of Total Thiol. First, $790 \mu \mathrm{l}$ of methanol and $150 \mu \mathrm{l}$ of Tris base-EDTA buffer were added to the test tube and then $50 \mu \mathrm{l}$ of homogenized tissue was added to it. After complete mixing, $50 \mu \mathrm{l}$ of DNTB reagent was added and vortexed again. The tubes were incubated for 15 minutes at room temperature and their OD was read at $412 \mathrm{~nm}$ [20].

\subsection{Determination of Antioxidant Enzymes}

2.7.1. Measurement of Superoxide Dismutase Activity. The SOD activity was measured based on its ability to inhibit the oxidation of NADPH. According to this method, the superoxide radical was formed during a chemical reaction of molecular oxygen in the presence of EDTA, manganese chloride, and mercaptoethanol (oxidation of NADPH depends on the availability of the superoxide anion). When SOD was added to the measurement mixture, nucleotide oxidation was prevented. Nucleotide oxidation changes at $340 \mathrm{~nm}$ at physiological $\mathrm{pH}$ were measured spectrophotometrically [21].

2.7.2. Measurement of Catalase Activity. Catalase activity was measured by Hugo Aebi method. In this method, the decomposition rate of $\mathrm{H}_{2} \mathrm{O}_{2}$ substrate at $420 \mathrm{~nm}$ was measured with a spectrophotometer [22].

2.8. Gene Expression of TNF- $\alpha, I L-1, T G F-\beta$, and $\alpha-S M A$. Real-time PCR calculated hepatic TNF-alpha, IL-1, $\alpha$-SMA, and TGF- $\beta$ mRNA. Total RNA was isolated from hepatic tissues using RNX Plus (Sinaclon, Tehran, Iran) and cDNA was generated on the basis of the manufacturer's technique from total RNA by cDNA Synthesis kit (Sinaclon, Tehran, Iran). RT-PCR was conducted using the instrument Rotor Gene 3000 (Bio-Rad, USA). The mRNA values of TNF- $\alpha$,
IL-1, $\alpha$-SMA, and TGF- $\beta$ were standardized to GAPDH. The PCR was completed in 40 cycles: TNF- $\alpha$ and IL- 1 at $95^{\circ} \mathrm{C}$ for 15 seconds, $58^{\circ} \mathrm{C}$ for 30 seconds, and $72^{\circ} \mathrm{C}$ for 30 seconds, $\alpha$-SMA at $95^{\circ} \mathrm{C}$ for 15 seconds, $63^{\circ} \mathrm{C}$ for 30 seconds, and $63^{\circ} \mathrm{C}$ for 30 seconds, and TGF- $\beta$ at $95^{\circ} \mathrm{C}$ for 15 seconds, $55^{\circ} \mathrm{C}$ for 30 seconds, $72^{\circ} \mathrm{C}$ for 30 seconds. Data of TNF- $\alpha$, IL- 1 , $\alpha$-SMA, and TGF- $\beta$ were calculated relative to GAPDH using the $2^{-\Delta \mathrm{Ct}}$ method.

2.9. Histological Examinations. For evaluation of alterations at the histological level, liver tissue portions of the right lobe were stored in 10 percent formalin. It was cleared with xylene after dehydration in the graded alcohol series. The tissues were embedded in paraffin sectioned and stained with haematoxylin and eosin reagent. The sections under a light microscope have been studied.

2.10. HPLC Analysis. An adept series gradient high-performance liquid chromatography (HPLC) (Knauer 2500 basic model) system was utilized for chromatographic analysis. The HPLC was equipped with a valve for the injection of sample with a $20 \mu \mathrm{L}$ sample loop, a detector of UV/ Vis, a quaternary pump, a per column (particle size of $5 \mu \mathrm{m}$, Eurospher 100-5 Cis), and in-line vacuum solvent degasser. The mobile phase consisted of water-acetonitrile with $\mathrm{v} / \mathrm{v} \%$ of $30: 70$ a flow rate $1.1 \mathrm{ml} \mathrm{min}^{-1}$. A wavelength of $220 \mathrm{~nm}$ was applied for the system, and the processes of separation and detection were carried out at ambient temperature.

2.11. Statistical Analysis. The obtained data were statistically analyzed using SPSS software (Version 16). First, the normality of data was determined by Kolmogorov-Smirnov test; then one-way analysis of variance was used for parametric data with Tukey post hoc test. The data were reported as mean \pm SEM. $P<0.05$ was reported as significant for all data.

\section{Results}

3.1. Biochemical Parameters. As presented in Figure 1, the level of AST, ALT, ALP, total bilirubin, and total protein increased significantly in the BDL group compared to SC-V group $(P<0.05)$. Treatment of OM extract at a dose of $300 \mathrm{mg} / \mathrm{kg}$ in the cholestatic group slightly decreased the content of AST, ALT, total bilirubin, and total protein as compared to the BDL-V group.

3.2. Hydroxyproline Content. As presented in Figure 2, the level of hepatic hydroxyproline increased in BDL-V rats compared to SC-V group $(P<0.05)$. Treatment of animals with OM significantly decreased hydroxyproline $(P<0.05)$.

3.3. Plasma Oxidative Stress Markers. Figure 3 shows that FRAP and MDA levels in the BDL-V group increased significantly compared to the SC-V group $(P<0.05)$, but our analysis showed that $\mathrm{OM}$ had no significant effect on plasma oxidative stress markers in the BDL groups. 


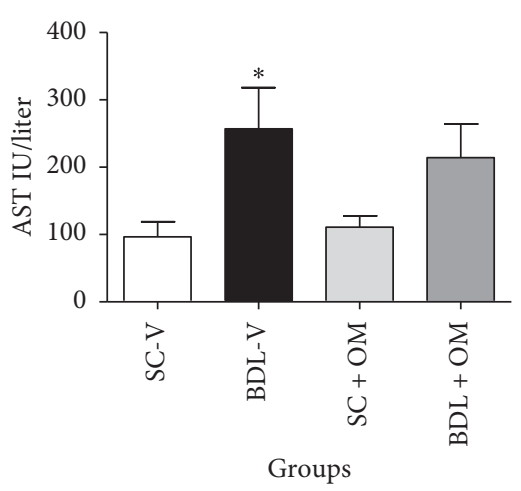

(a)

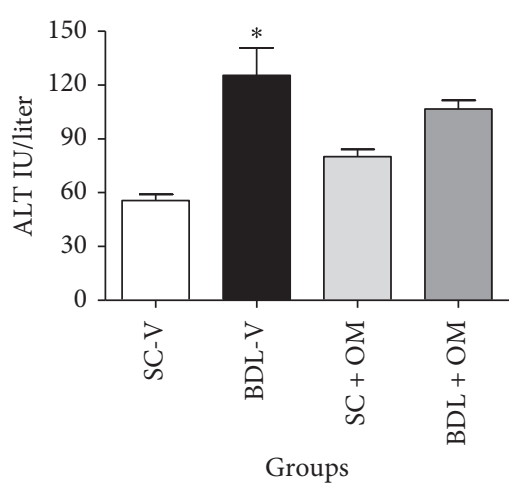

(b)

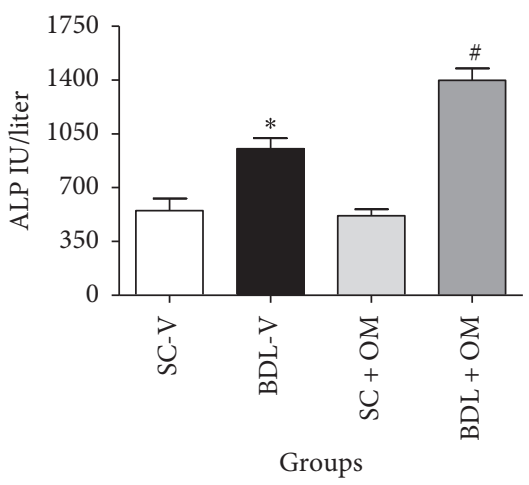

(c)

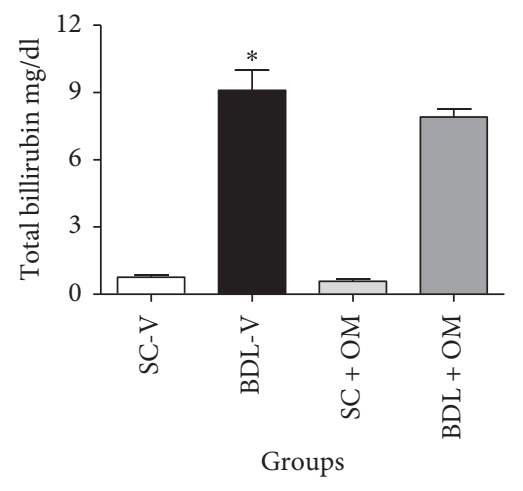

(d)

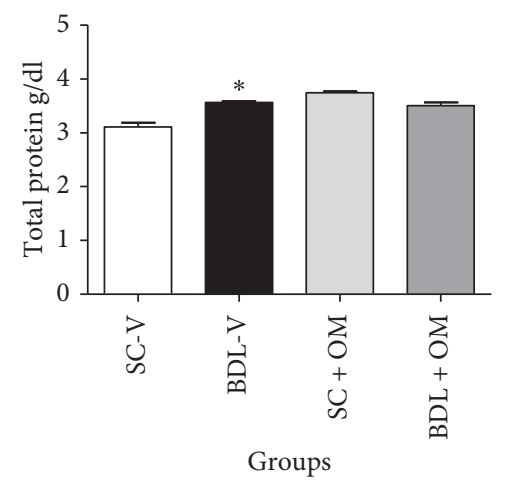

(e)

Figure 1: Effect of OM extract on biochemical markers. Each value represents the mean \pm SEM. ${ }^{*}$ Significantly different from SC-V, $P$ value $\leq 0.05$. \# Significantly different from BDL-V, $P$ value $\leq 0.05$. AST, aspartate aminotransferase (a); ALT, alanine aminotransferase (b); ALP, alkaline phosphatase (c); total bilirubin (d); total protein (e); SC-V, sham control + vehicle; BDL-V, bile duct-ligation + vehicle; OM, Origanum majorana.

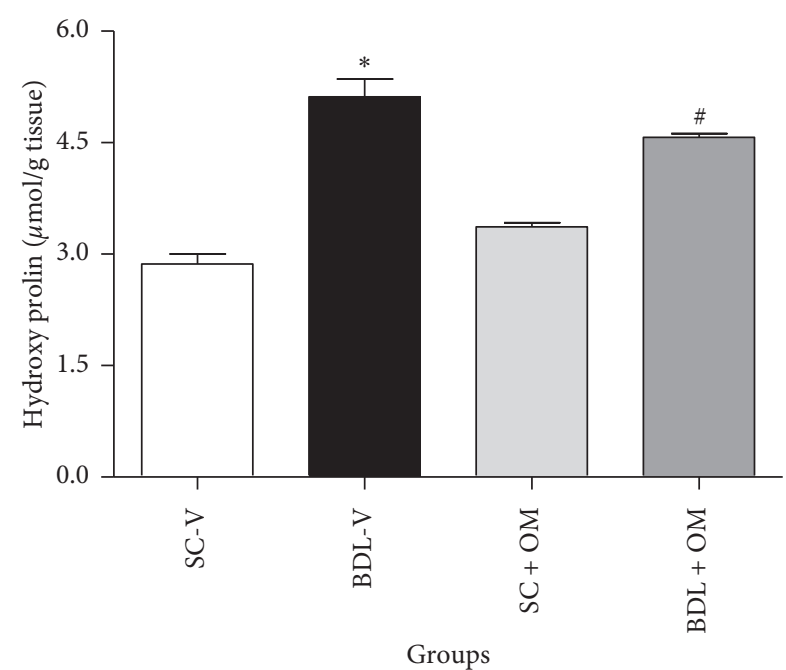

FIGURE 2: Effect of OM on hydroxyproline level. Each value represents the mean \pm SEM. ${ }^{*}$ Significantly different from $S C-V, P$ value $\leq 0.05$. ${ }^{\#}$ Significantly different from BDL-V, $P$ value $\leq 0.05$. SC-V, sham control + vehicle; BDL-V, bile duct-ligation + vehicle; OM, Origanum majorana.

3.4. Liver Oxidative Stress Markers. As illustrated in Figure 4 FRAP and MDA levels did not change in the BDL-V group compared to the SC-V group. TSH level in the BDL-V group significantly decreased as compared to the SC-V group $(P<0.05)$. Our finding also showed that OM at a dose of $300 \mathrm{mg} / \mathrm{kg}$ had no significant effect on the FRAP, TSH, and MDA levels as compared to the BDL-V group.

3.5. Antioxidants Enzymes. As shown in Figure 5, CAT and SOD activity did not alter in the BDL-V group compared to the SC-V group. Administration of OM at a dose of $300 \mathrm{mg} /$ $\mathrm{kg}$ caused a significant increase in CAT activity in the BDL group in contrast to the BDL-V group but it had no significant effect on SOD activity.

3.6. Histopathological Examination. Histological studies of $\mathrm{SC}-\mathrm{V}$ and $\mathrm{SC}+\mathrm{OM}$ groups revealed that the liver had a normal hepatic lobule structure (Figures 6(a) and 6(c)). Hepatic sections of BDL-V rats showed activation of hepatocytes and areas of hepatic necrosis (Figure 6(b)). Also, the liver of BDL rats treated with OM did not show significant improvement in the histopathological changes (Figure 6(d)). Examination of liver tissue showed that bile duct proliferation and inflammation increased significantly $(80 \%)$ in the BDL group compared to the SC-V rats $(P<0.05)$, and $\mathrm{OM}$ had no effect on inflammation score of hepatic tissue (Figure 7). 


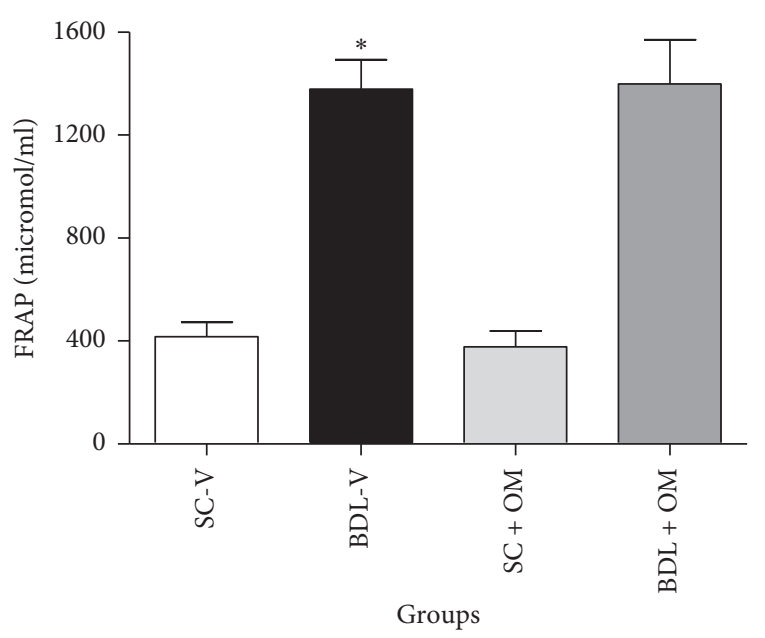

(a)

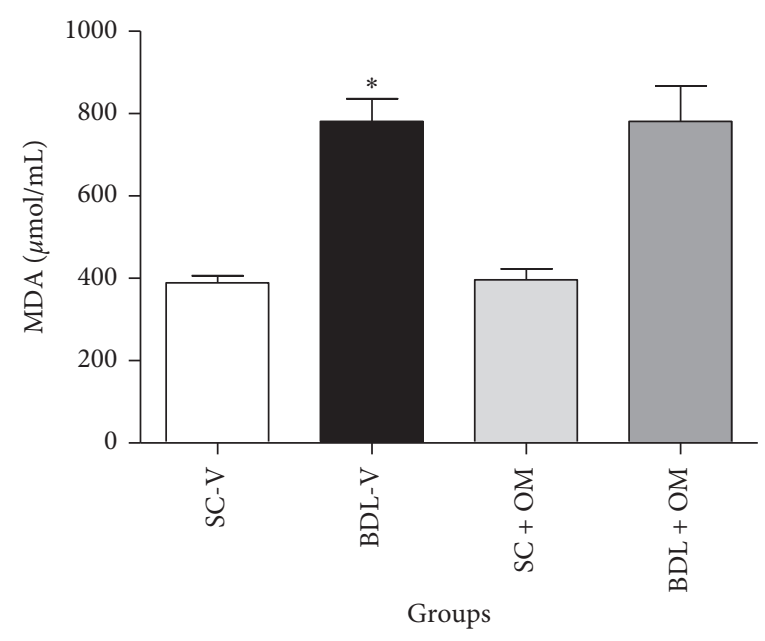

(b)

Figure 3: Effect of OM on FRAP (a) and MDA (b) plasma levels. Each value represents the mean \pm SEM. *Significantly different from SC, $P$ value $\leq 0.05$. FRAP, ferric reducing antioxidant power; MDA, malondialdehyde; SC-V, sham control + vehicle; BDL-V, bile ductligation + vehicle; OM, Origanum majorana.

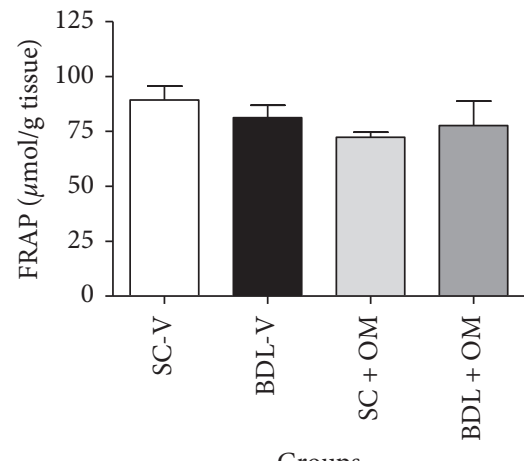

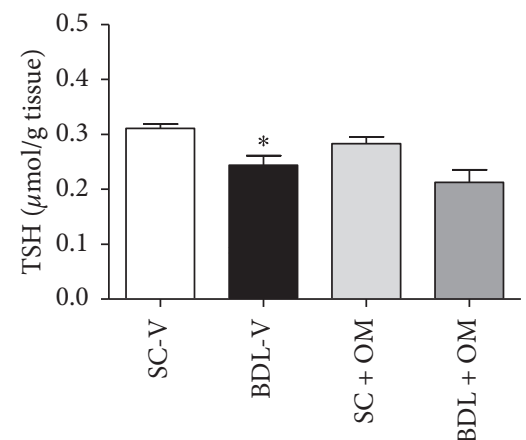

Groups

(b)

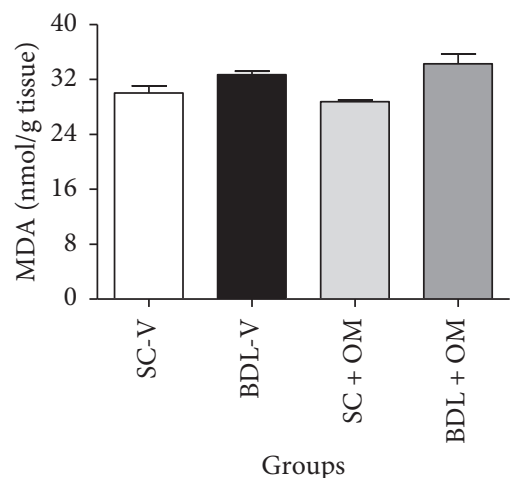

(c)

FIgURE 4: Effect of OM on the tissue oxidative stress markers. FRAP, ferric reducing activity of plasma. (a) TSH, total thiol. (b) MDA, malondialdehyde. (c) Each value represents the mean \pm SEM. * Significantly different from SC-V, $P$ value $\leq 0.05$. SC-V, sham control + vehicle; BDL-V, bile duct-ligation + vehicle. OM, Origanum majorana.

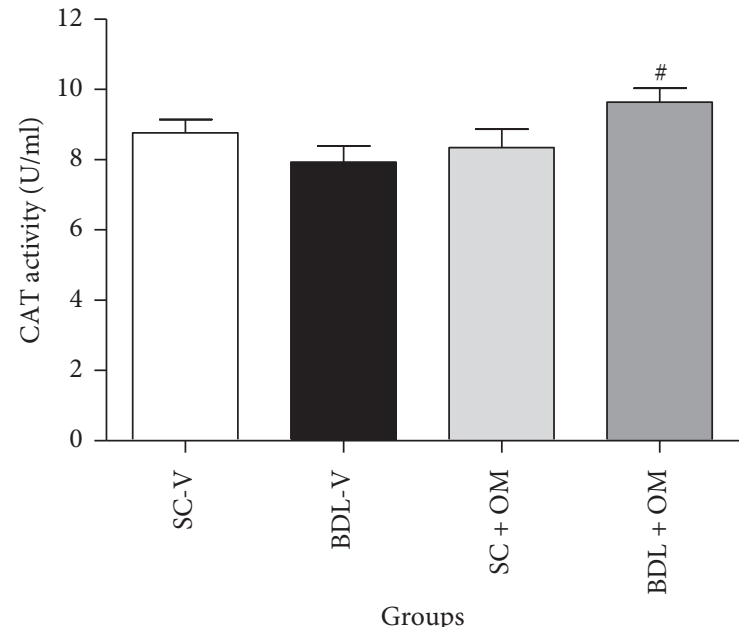

(a)

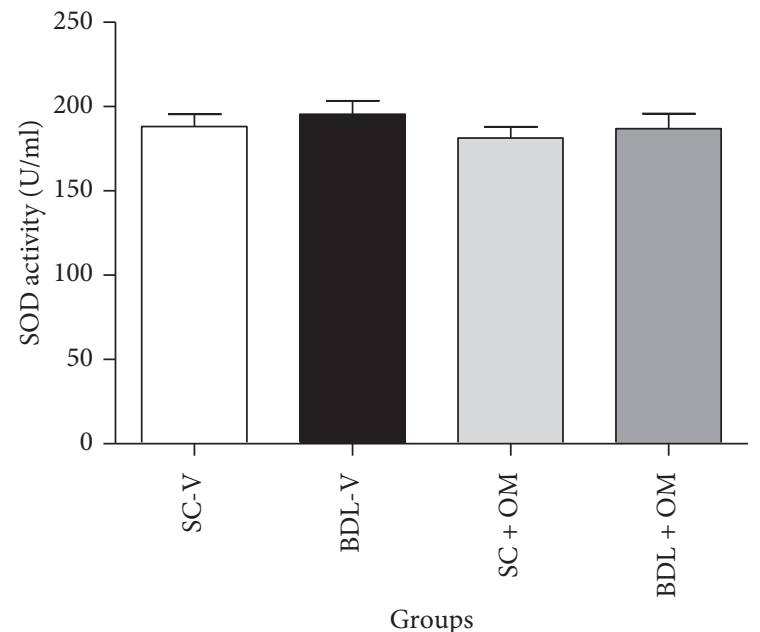

(b)

FIGURE 5: Effect of OM on CAT (a), SOD (b) activity in the liver tissue. Each value represents the mean \pm SEM. SC-V, sham control + vehicle; $\mathrm{BDL}-\mathrm{V}$, bile duct-ligation + vehicle; OM, Origanum majorana; CAT, catalase; SOD, superoxide dismutase. ${ }^{\#}$ Significantly different from BDL-V group, $P \leq 0.05$. 


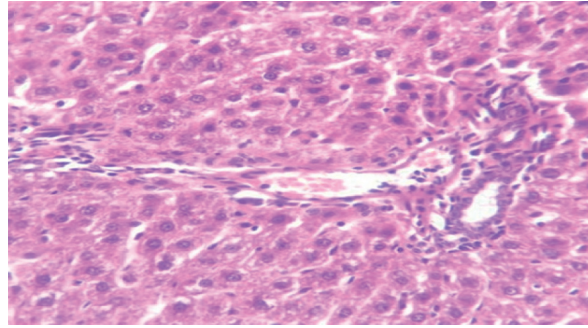

(a)

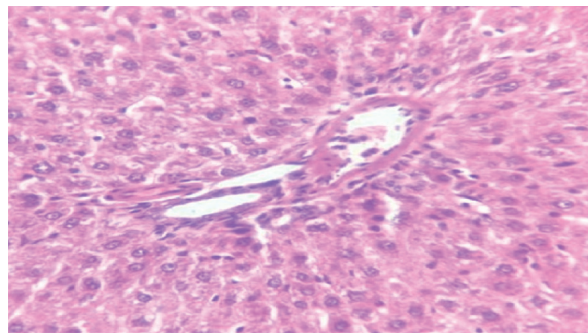

(c)

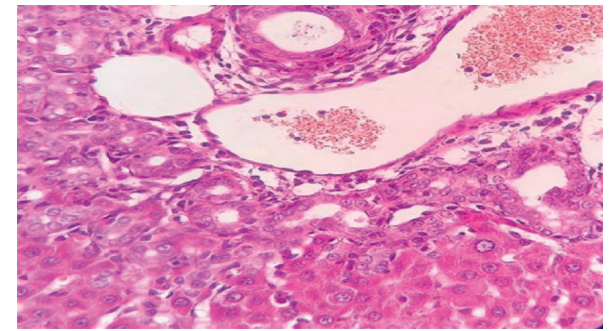

(b)

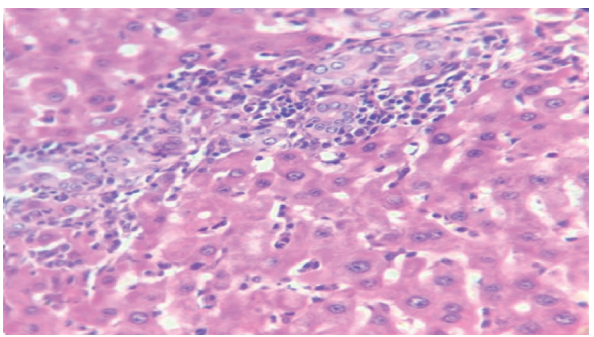

(d)

FIgURE 6: Liver section of the SC-V group (a), BDL-V group (b), SC+ OM group (c), and BDL + OM group (d).

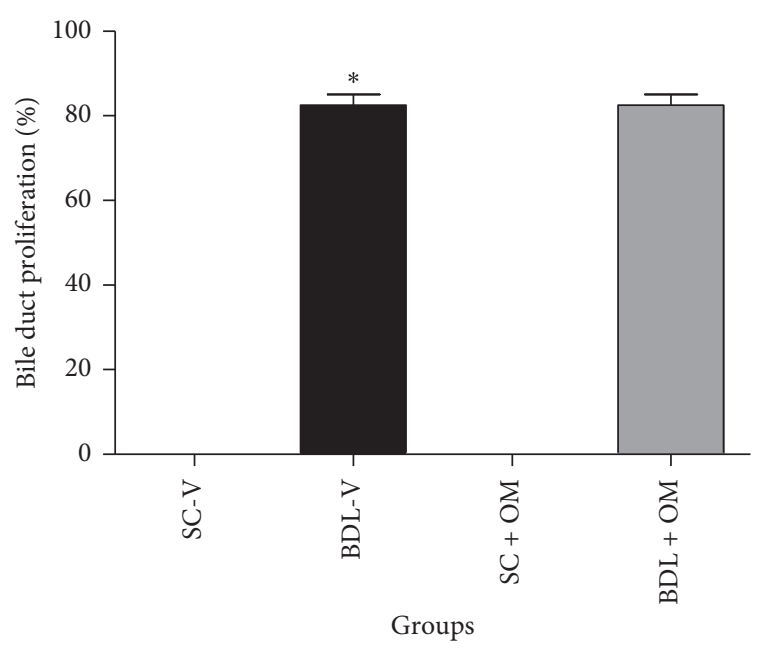

(a)

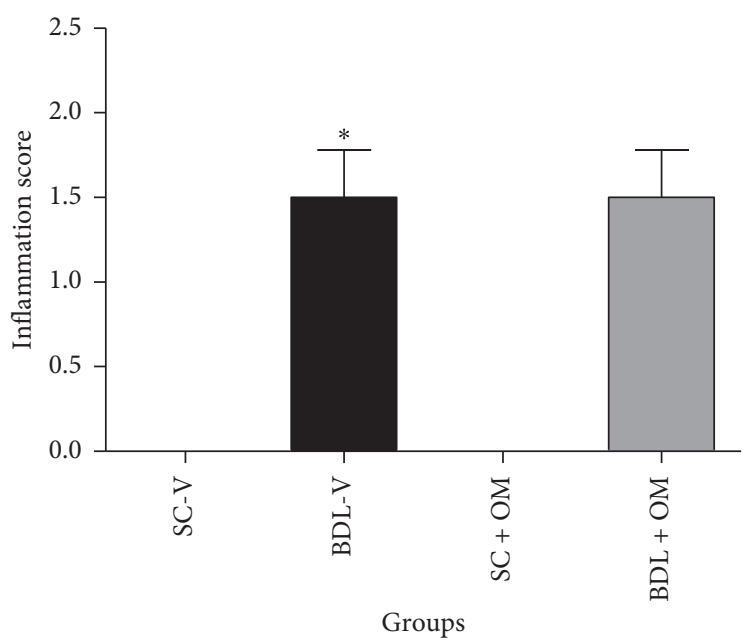

(b)

FIGURE 7: Effect of OM on bile duct proliferation (a), and inflammation of hepatic tissue (b). SC-V, sham control + vehicle; BDL-V, bile duct-ligation + vehicle and OM, Origanum majorana. ${ }^{*}$ Significantly different from SC-V groups, $P \leq 0.05$. ${ }^{\#}$ Significantly different from BDL-V group, $P \leq 0.05$.

3.7. Gene Expression of TNF- $\alpha, I L-1, \alpha-S M A$, and TGF- $\beta$. The mRNA levels of TNF- $\alpha$, IL- $1, \alpha$-SMA, and TGF- $\beta$ significantly elevated in the liver tissue of BDL-V group in contrast to $\mathrm{SC}-\mathrm{V}$ rats $(P \leq 0.05)$. Our results indicated that $\mathrm{OM}$ at a dose of $300 \mathrm{mg} / \mathrm{kg}$ had no significant effect on expression of TNF- $\alpha$, IL- 1 , and $\alpha$-SMA levels against the BDL- $\mathrm{V}$ rats, but significantly reduced the relative expression of TGF- $\beta$ against the BDL-V rats $(P \leq 0.05)$ (Figure 8 ).

3.8. HPLC Analysis. Typical analysis of the standard carvacrol in aqueous solution, related calibration curve, and methanolic extraction of OM collected at $8 \mathrm{~min}$ are shown in
Figure 9(a). The calibration curve (Figure $9(\mathrm{~b})$ ) was prepared with carvacrol and was found to be linear $\left(R^{2}=0.9988\right)$ in the concentration range $\left(0.10-2 \mathrm{mg} \mathrm{L}^{-1}\right)$ used. Based on the HPLC analysis, the concentration of carvacrol on the extract of $\mathrm{OM}$ was $33.65 \pm 1.87 \mathrm{mg} \mathrm{g}^{-1}$ (Figure 9(c)).

\section{Discussion}

Hepatic cholestasis is one of the causes of liver cirrhosis and fibrosis. Nowadays, researchers are trying to understand the mechanisms associated with liver disorders. BDL is a well-known laboratory model that mimics the complex mechanisms of liver cirrhosis. The in vitro model of bile 


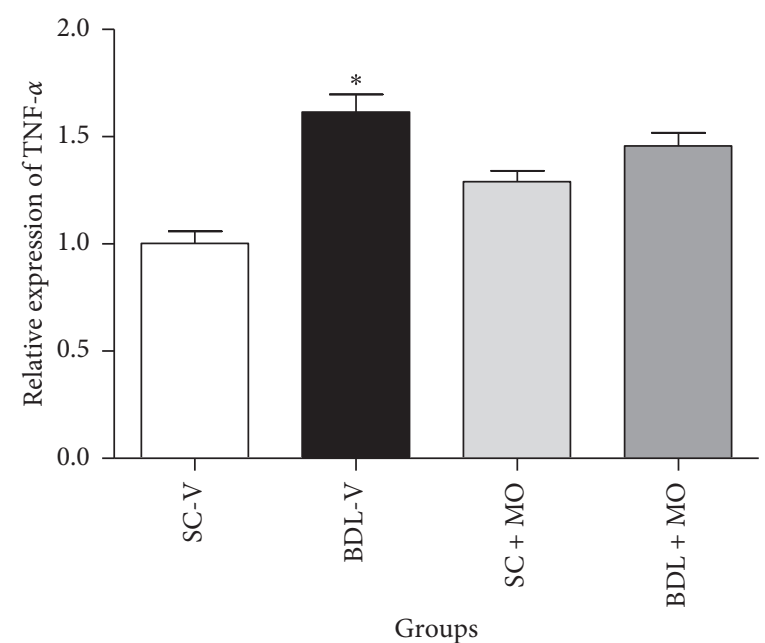

(a)

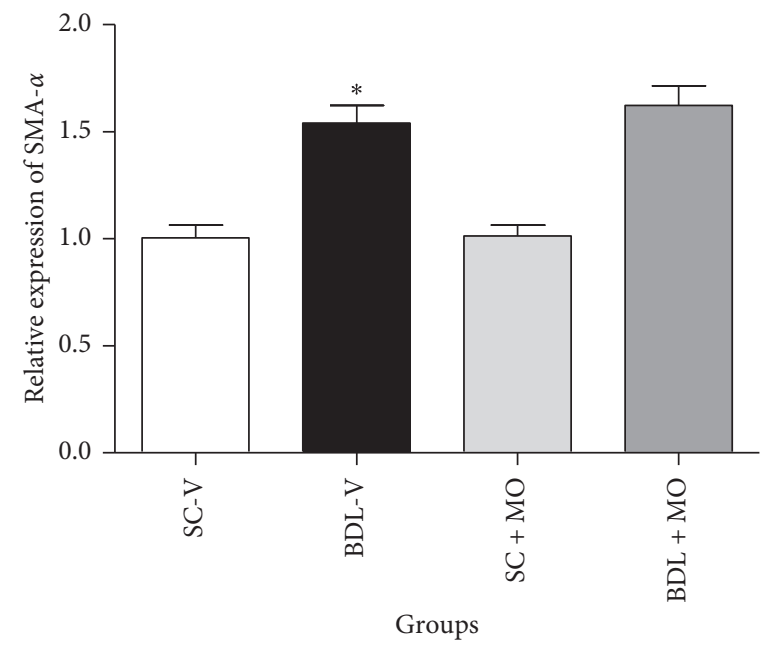

(c)

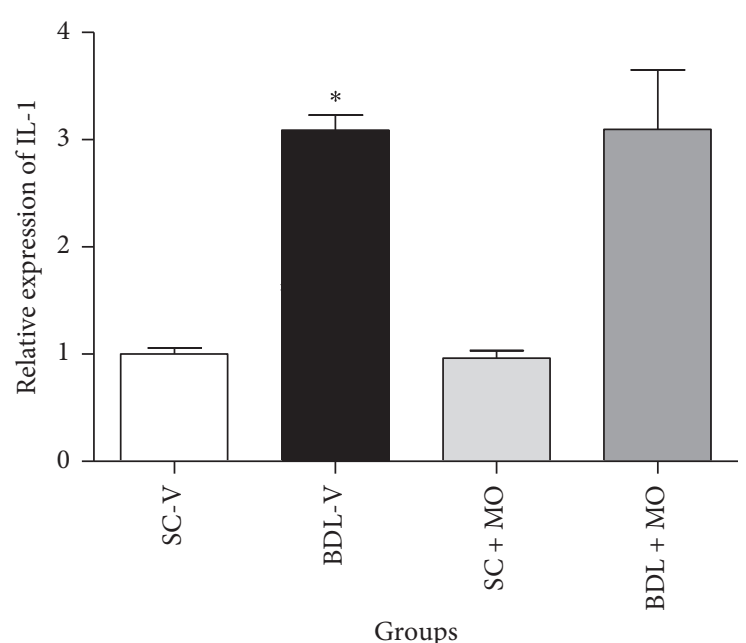

(b)

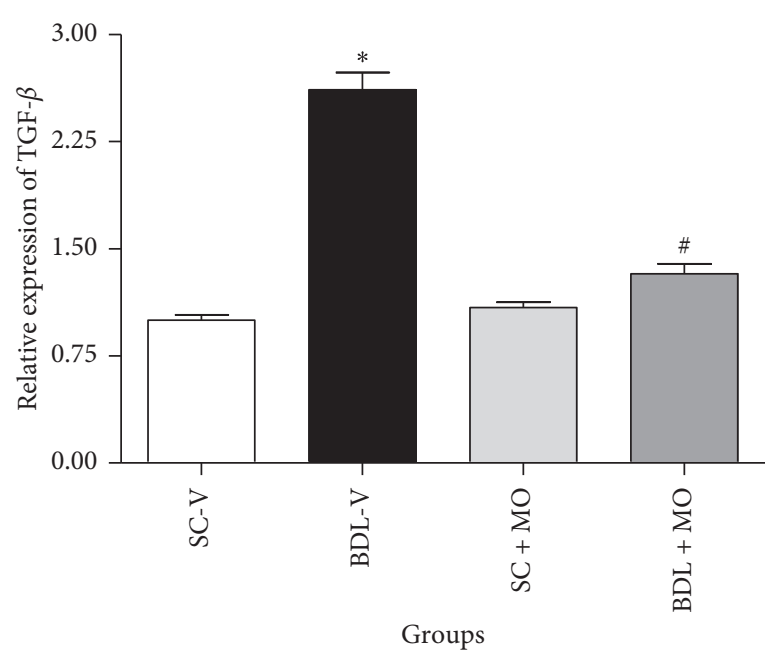

(d)

Figure 8: The effect of OM on the mRNA levels of TNF- $\alpha$, IL-1, TGF- $\beta$, and $\alpha$-SMA in BDL-induced cholestatic rats. SC-V, sham control + vehicle; BDL-V, bile duct-ligation + vehicle and OM, Origanum majorana. ${ }^{*}$ Significantly different from SC-V groups, $P \leq 0.05$. \# Significantly different from BDL-V group, $P \leq 0.05$.

duct ligation in rodents has been used as an experimental method in research for many years. The first protocol for this model was proposed more than three decades ago in which cannulation, occlusion, and ligation cause cirrhosis with changes in liver tissue of rodents that became similar to those seen in human cirrhosis [23]. This animal model causes apoptosis and necrosis of liver cells and ultimately liver fibrosis and cirrhosis through bile cytotoxic components such as bile lipophilic acid and also responsible for plasma membrane damage and oxidative stress. In this study, we evaluated the protective and antioxidant effects of $\mathrm{OM}$ in BDL rats and analyzed the role of this plant in expressing the liver genome in these rats.

The serum levels of AST, ALT, and ALP are important indexes for the clinical manifestation of cholestasis/cirrhosis. As expected, according to Tahan et al. study [24], the level of biochemical markers including AST, ALT, ALP, and total bilirubin increased significantly in the BDL-V group compared to the SC-V group. AST and ALT mainly distributed in the hepatocytes; they release into the blood circulation when hepatocytes membrane are damaged by bile duct obstruction; then these changes in liver enzymes cause loss of liver tissue integrity and cell death $[25,26]$. In our study, OM treatment slightly decreased the elevated level of AST and ALT induced by BDL. The results suggested that OM extract, due to its antioxidant properties, slightly reduces liver damage by inhibiting the formation of free radicals, thus preventing the release of liver enzymes into the blood.

One of the standard indicators for assessing collagen formation in liver tissue is the measurement of hydroxyproline levels. In a study by Zhao et al., hydroxyproline level in the BDL-V group was higher than the SC-V group [27]. Our findings also showed a significant increase in hydroxyproline level in the BDL-V group compared to SCV. In the present study, the hydroxyproline content in the 


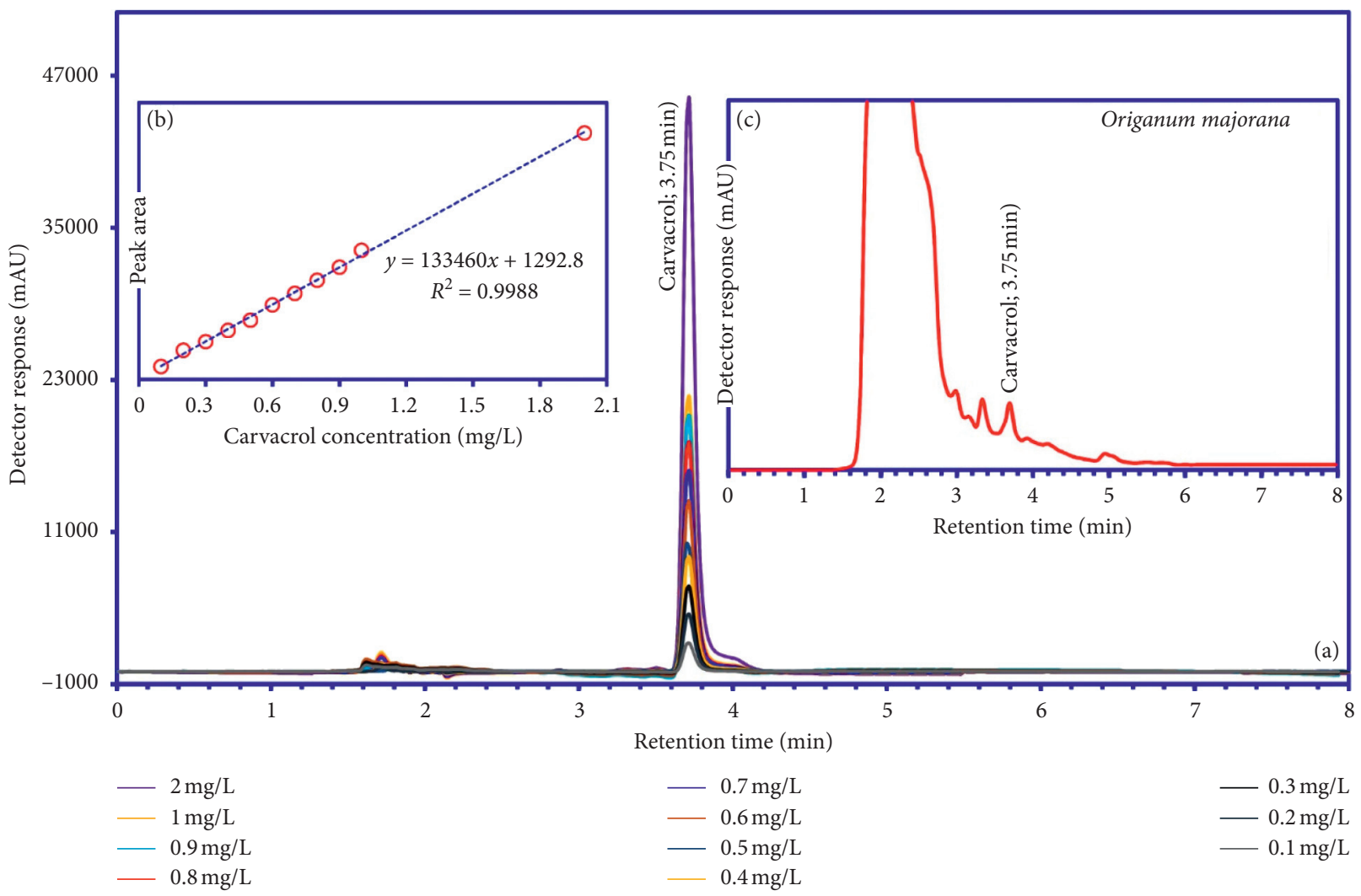

FIGURE 9: HPLC analysis of carvacrol in the methanolic extraction of Origanum majorana.

liver of $\mathrm{BDL}$ rats receiving $\mathrm{OM}$ extract was significantly lower than the BDL-V group $(P<0.05)$. Accumulation of bile acids damages hepatocytes; then, inflammatory cells produce inflammatory cytokines and ROS, leading to inflammatory responses and collagen deposition. Presumably, OM hydroalcoholic extract reduces collagen production by trapping ROS, thereby reducing the hydroxyproline content in the liver tissue.

An imbalance between oxidants and the antioxidant system in cells causes oxidative stress. Oxidative stress and related events are the most important mechanisms associated with cholestasis/cirrhosis. BDL not only increased free radicals but also decreased antioxidant activity [28]. High level of MDA indicates an increase in lipid peroxidation, which ultimately causes tissue damage as well as the overformation of free radicals by impairing the antioxidant defense mechanism $[29,30]$. According to the study of Zhao et al., MDA levels increased in both serum and liver tissue in the BDL group [27]. Fang et al. indicated a significant increase in MDA level in BDL-induced cholestasis rats compared with the SC group [31]. The present data showed that the plasma MDA of cholestatic rats significantly increased compared to the SC-V group, and OM had no effect on plasma MDA levels.

FRAP represents the antioxidative capacity of plasma [20]. In the present study, the plasma FRAP level of the BDL$\mathrm{V}$ group had a significant increase compared to SC-V group. In 2020, Ghaeni et al. found that OM extract significantly increased FRAP in the high-fat diet induced hepatosteatosis
[32]. FRAP mostly supports the antioxidant activity of OM plant, which is mostly due to its phenolic compounds [33]. Our findings showed that FRAP levels in the BDL + OM group slightly increased compared to BDL-V animals. This difference may be due to the lower dose of OM used in our study.

Total thiols contain endogenous antioxidant compounds that also have radical scavenging effects and metal chelators, which, for this reason, has played an important role against oxidative stress [34]. According to a study by Sadeghi et al. in 2019, our results showed that the level of TSH in the BDL-V group significantly decreased as compared to the SC-V group [20], whereas administration of OM had no significant effect on the level of TSH compared to the BDL-V group.

It has been reported that the administration of medicinal plants and natural compounds with antioxidant activity such as hydroalcoholic extract of Watercress [20], Ziziphus mauritiana leaf [35], polydatin [31], quercetin [36], and Marjoram oil [37] can protect the liver against oxidative damage caused by cholestasis. SOD and CAT are antioxidants that prevent the production of free radicals and also work as part of the first line of defense in the living cells [30]. Zhao et al. showed that SOD activity was significantly increased in the OM treated groups compared with the control group [27]. Other studies have reported that Origanum vulgare consumption significantly increases the activity of the antioxidant enzymes such as SOD and CAT $[33,38]$. Our results showed that the SOD activity did not change in all 
groups but CAT activity significantly increased in the $\mathrm{BDL}+\mathrm{OM}$ group as compared to only BDL-V rats. CAT is enriched in liver tissue which is responsible for catalyzing the decomposition of hydrogen peroxide to water and oxygen. It seems that phenolic compounds stimulate CAT activity which reduces the number of free radicals, suggesting that $\mathrm{OM}$ has the antioxidant effects on the cholestatic liver.

One of the important elements in the onset and progression of liver fibrosis is inflammation and immune responses. Inflammatory cells are a significant source of cytokines that can initially mediate inflammatory responses and later cause tissue fibrosis [39]. TNF- $\alpha$ and TGF- $\beta$ as proinflammatory cytokines signal the expression of several inflammatory mediators and the regulation of the inflammatory response, which are increased by oxidative stress $[40,41]$. Recent findings showed that hepatic TNF- $\alpha$ and TGF- $\beta$ genes expression was increased in liver injury $[36,42]$, which is consistent with our results. In our work, OM extract reduced hepatic TGF- $\beta$ gene expression, which was increased by BDL-V. However, TNF- $\alpha$ expression was not significantly different from BDL-V. TGF- $\beta$ is a profibrotic cytokine that increases the inhibition of metalloproteinases, thereby reducing the degradation of the extracellular matrix and increasing collagen synthesis. Accordingly, OM extract may suppress the inflammatory response in rats by inhibiting the proinflammatory marker (TGF- $\beta$ ) and/or controlling the oxidant status.

\section{Conclusion}

The data of the present study showed that oral treatment with OM $(300 \mathrm{mg} / \mathrm{kg})$ produced a moderate hepatoprotective and inflammatory activity in the cholestatic rats. In fact, to confirm this finding, further studies with different doses of extracts are needed.

\section{Data Availability}

The data supporting the findings of this study are available within the article.

\section{Conflicts of Interest}

There are no conflicts of interest among the authors.

\section{Acknowledgments}

This work was supported by council of research of Yasuj University of Medical Sciences, Yasuj, Iran. Financially, this research was supported by Research Vice-Chancellery of Yasuj University of Medical Sciences, I.R. Iran (grant number: 980036).

\section{References}

[1] A. H. Doustimotlagh, E. P. Kokhdan, H. Vakilpour et al., "Protective effect of Nasturtium officinale R. Br and quercetin against cyclophosphamide-induced hepatotoxicity in rats," Molecular Biology Reports, vol. 47, no. 7, pp. 5001-5012, 2020.
[2] M. S. Padda, M. Sanchez, A. J. Akhtar, and J. L. Boyer, "Druginduced cholestasis," Hepatology, vol. 53, no. 4, pp. 1377-1387, 2011.

[3] E. Izadpanah, K. Hassanzadeh, V. Yousefinejad, K. Shahveisi, N. Fatahi, and M. R. Moloudi, "Effect of selegiline on liver cholestasis induced by bile duct ligation in rat," Scientific Journal of Kurdistan University of Medical Sciences, vol. 21, no. 5, pp. 20-30, 2016.

[4] W.-r. Cao, J.-q. Ge, X. Xie et al., "Protective effects of petroleum ether extracts of Herpetospermum caudigerum against $\alpha$-naphthylisothiocyanate-induced acute cholestasis of rats," Journal of Ethnopharmacology, vol. 198, pp. 139-147, 2017.

[5] S. I. Ramadan, M. Shalaby, N. Afifi, and H. El-Banna, "Hepatoprotective and antioxidant effects of Silybum marianum plant in rats," International Journal for Agro Veterinary and Medical Sciences, vol. 5, pp. 541-547, 2011.

[6] M. Kunz, C. S. Gama, A. C. Andreazza et al., "Elevated serum superoxide dismutase and thiobarbituric acid reactive substances in different phases of bipolar disorder and in schizophrenia," Progress in Neuro-Psychopharmacology and Biological Psychiatry, vol. 32, no. 7, pp. 1677-1681, 2008.

[7] P. Gałecki, J. Szemraj, M. Bieńkiewicz, A. Florkowski, and E. Gałecka, "Lipid peroxidation and antioxidant protection in patients during acute depressive episodes and in remission after fluoxetine treatment," Pharmacological Reports :PR, vol. 61, no. 3, pp. 436-447, 2009.

[8] A. Soliman, S. Desouky, M. Marzouk, and A. Sayed, "Origanum majorana attenuates nephrotoxicity of cisplatin anticancer drug through ameliorating oxidative stress," Nutrients, vol. 8, no. 5, p. 264, 2016.

[9] S. Desouky, M. Marzouk, A. M. Soliman, and A. A. Sayed, "Modulatory effect of Origanum majorana extract against cisplatin-induced dyslipidemia in rats," International Journal of Current Life Science, vol. 4, no. 6, pp. 228-234, 2015.

[10] N. Benhalilou, H. Alsamri, A. Alneyadi, K. Athamneh, A. Alrashedi, and N. Altamimi, "Origanum majorana ethanolic extract promotes colorectal cancer cell death by triggering abortive autophagy and activation of the extrinsic apoptotic pathway," Frontiers in Oncology, vol. 9, p. 795, 2019.

[11] S. Duletić-Laušević, A. A. Aradski, S. Kolarević, B. VukovićGačić, M. Oalđe, and J. Živković, "Antineurodegenerative, antioxidant and antibacterial activities and phenolic components of Origanum majorana L.(Lamiaceae) extracts," Journal of Applied Botany and Food Quality, vol. 91, pp. 126-134, 2018.

[12] A. El-Ghany and Y. Nanees, "Effect of marjoram leaves on injured liver in experimental rats," Report and Opinion, vol. 2, no. 12, pp. 181-191, 2010.

[13] K. K. Kaur, G. Allahbadia, and M. Singh, "Rosmarinic acid-a new hope for liver diseases like cirrhosis, hepatocellular carcinoma-needs translation to humans," EC Endocrinology and Metabolic Research, vol. 4, pp. 289-301, 2019.

[14] W.-Y. Chen, C.-J. Chen, J.-W. Liao, and F. C. Mao, "Chromium attenuates hepatic damage in a rat model of chronic cholestasis," Life Sciences, vol. 84, no. 17-18, pp. 606-614, 2009.

[15] Z. Ackerman, M. Weinreb, G. Amir, and R. D. Pollak, "Bone mineral metabolism and histomorphometry in rats with cholestatic liver disease," Liver, vol. 22, no. 2, pp. 166-172, 2002.

[16] N. Afifi, M. Shalaby, H. eLbanna, and S. Ramadan, "Evaluation of the antioxidant activity of Marjoram plant (Origanium Majorana L.) in CCl 4-intoxicated rats," Spatula DD - Peer Reviewed 
Journal on Complementary Medicine and Drug Discovery, vol. 4, no. 1, pp. 33-40, 2014.

[17] M. Nadimi, Z. Mohammadali, and M. Madani, "The effect of aqueous and ethanolic extracts of Teucrium polium on Candida albicans and two species of Malassezia," 2013.

[18] A. H. Doustimotlagh, A. R. Dehpour, S. Etemad-Moghadam, M. Alaeddini, Y. Kheirandish, and A. Golestani, "Nitrergic and opioidergic systems affect radiographic density and histomorphometric indices in bile-duct-ligated cirrhotic rats," Histology and Histopathology, vol. 32, no. 7, pp. 743-749, 2017.

[19] I. F. F. Benzie and J. J. Strain, "The ferric reducing ability of plasma (FRAP) as a measure of "antioxidant power": the FRAP assay," Analytical Biochemistry, vol. 239, no. 1, pp. 70-76, 1996.

[20] H. Sadeghi, N. Azarmehr, F. Razmkhah et al., "The hydroalcoholic extract of watercress attenuates protein oxidation, oxidative stress, and liver damage after bile duct ligation in rats," Journal of Cellular Biochemistry, vol. 120, no. 9, pp. 14875-14884, 2019.

[21] F. Paoletti and A. Mocali, "[18] Determination of superoxide dismutase activity by purely chemical system based on NAD(P)H oOxidation," Oxygen Radicals in Biological Systems Part B: Oxygen Radicals and Antioxidants, vol. 186, pp. 209-220, 1990.

[22] H. Aebi, "[13] Catalase in vitro," Methods in Enzymology, vol. 105, pp. 121-126, 1984.

[23] C. G. Tag, S. Sauer-Lehnen, S. Weiskirchen, E. BorkhamKamphorst, R. H. Tolba, and F. Tacke, "Bile duct ligation in mice: induction of inflammatory liver injury and fibrosis by obstructive cholestasis," Journal of Visualized Experiments: JoVE, vol. 96, 2015.

[24] G. Tahan, O. Tarcin, V. Tahan et al., "The effects of N-acetylcysteine on bile duct ligation-induced liver fibrosis in rats," Digestive Diseases and Sciences, vol. 52, no. 12, pp. 3348-3354, 2007.

[25] P. M. Amália, M. N. Possa, M. C. Augusto, and L. S. Francisca, "Quercetin prevents oxidative stress in cirrhotic rats," $D i$ gestive Diseases and Sciences, vol. 52, no. 10, pp. 2616-2621, 2007.

[26] N. El-Lakkany, S. Seif el-Din, A.-N.-A. Sabra, O. Hammam, and F.-L. Ebeid, "Co-administration of metformin and $\mathrm{N}$-acetylcysteine with dietary control improves the biochemical and histological manifestations in rats with nonalcoholic fatty liver," Research in Pharmaceutical Sciences, vol. 11 , no. 5 , p. $374,2016$.

[27] S.-S. Zhao, N.-R. Li, W.-L. Zhao et al., "D-chiro-inositol effectively attenuates cholestasis in bile duct ligated rats by improving bile acid secretion and attenuating oxidative stress," Acta Pharmacologica Sinica, vol. 39, no. 2, pp. 213-221, 2018.

[28] M. Mansourian, A. Mirzaei, N. Azarmehr, H. Vakilpour, E. P. Kokhdan, and A. H. Doustimotlagh, "Hepatoprotective and antioxidant activity of hydroalcoholic extract of Stachys pilifera. benth on acetaminophen-induced liver toxicity in male rats," Heliyon, vol. 5, no. 12, Article ID e03029, 2019.

[29] C. Aktas, M. Kanter, M. Erboga, R. Mete, and M. Oran, "Melatonin attenuates oxidative stress, liver damage and hepatocyte apoptosis after bile-duct ligation in rats," Toxicology and Industrial Health, vol. 30, no. 9, pp. 835-844, 2014.

[30] H. Saleh, A. M. Soliman, A. S. Mohamed, and M. A. Marie, "Antioxidant effect of sepia ink extract on extrahepatic cholestasis induced by bile duct ligation in rats," Biomedical and Environmental Sciences: BES, vol. 28, no. 8, pp. 582-594, 2015.

[31] J. Fang, L. Luo, Z. Ke et al., "Polydatin protects against acute cholestatic liver injury in mice via the inhibition of oxidative stress and endoplasmic reticulum stress," Journal of Functional Foods, vol. 55, pp. 175-183, 2019.

[32] A. Ghaeni Pasavei, R. Mohebbati, N. Boroumand, A. Ghorbani, A. Hosseini, and S. Taraz Jamshidi, "Antihypolipidemic and anti-oxidative effects of hydroalcoholic extract of origanum majorana on the hepatosteatosis induced with high-fat diet in rats," Malaysian Journal of Medical Sciences, vol. 27, no. 1, 2020.

[33] A. G. Pasavei, R. Mohebbati, N. Boroumand, A. Ghorbani, A. Hosseini, and S. T. Jamshidi, "Anti-hypolipidemic and anti-oxidative effects of hydroalcoholic extract of origanum majorana on the hepatosteatosis induced with high-fat diet in rats," The Malaysian Journal of Medical Sciences: MJMS, vol. 27, no. 1, p. 57, 2020.

[34] D. Terzioglu, L. Uslu, G. Simsek et al., "The effects of hyperbaric oxygen treatment on total antioxidant capacity and prolidase activity after bile duct ligation in rats," Journal of Investigative Surgery, vol. 30, no. 6, pp. 376-382, 2017.

[35] D. Dahiru and O. Obidoa, "Evaluation of the antioxidant effects of Ziziphus mauritiana lam. leaf extracts against chronic ethanol-induced hepatotoxicity in rat liver," African Journal of Traditional, Complementary and Alternative Medicines, vol. 5, no. 1, pp. 39-45, 2008.

[36] N. Nassef, E. Abu-Shadi, S. El Agaty, and G. Abdel Hamid, "Quercetin mitigates liver injury in a rat model of liver cholestasis," Bulletin of Egyptian Society for Physiological Sciences, vol. 40, no. 1, pp. 84-95, 2020.

[37] N. A. A. Aita and F. F. Mohammed, "Effect of marjoram oil on the clinicopathological, cytogenetic and histopathological alterations induced by sodium nitrite toxicity in rats," Global Vet, vol. 12, pp. 606-616, 2014.

[38] H. Daghigh Kia, R. Farhadi, I. Ashrafi, and M. Mehdipour, "Anti-oxidative effects of ethanol extract of Origanum vulgare on kinetics, microscopic and oxidative parameters of cryopreserved Holstein bull spermatozoa," Iranian Journal of Applied Animal Science, vol. 6, no. 4, pp. 783-789, 2016.

[39] A. J. Czaja, "Hepatic inflammation and progressive liver fibrosis in chronic liver disease," World Journal of Gastroenterology, vol. 20, no. 10, p. 2515, 2014.

[40] K. Cheng, N. Yang, and R. I. Mahato, "TGF- $\beta 1$ gene silencing for treating liver fibrosis," Molecular Pharmaceutics, vol. 6, no. 3, pp. 772-779, 2009.

[41] R. Krithika, V. Jyothilakshmi, and R. J. Verma, "Phyllanthin inhibits CCl4-mediated oxidative stress and hepatic fibrosis by down-regulating TNF- $\alpha / \mathrm{NF}-\kappa \mathrm{B}$, and pro-fibrotic factor TGF- $\beta 1$ mediating inflammatory signaling," Toxicology and Industrial Health, vol. 32, no. 5, pp. 953-960, 2016.

[42] A. Sharifi-Rigi, E. Heidarian, and S. A. Amini, "Protective and anti-inflammatory effects of hydroalcoholic leaf extract of Origanum vulgare on oxidative stress, TNF- $\alpha$ gene expression and liver histological changes in paraquat-induced hepatotoxicity in rats," Archives of Physiology and Biochemistry, vol. 125, no. 1, pp. 56-63, 2019. 